\title{
Interference effects within the kindling paradigm
}

\author{
JOHN GAITO \\ York University, Downsview, M3J 1P3, Ontario, Canada
}

\begin{abstract}
There are three types of interference effects during kindling: that produced by alternate stimulation of homologous brain sites, by successive stimulation of one site, and by stimulation of one site by different frequencies. These three types of interference appear to be similar. Facilitation and interference effects during kindling seem to be generated by the operation of two factors: a "neurological trace" process, possibly involving synaptic changes, and an "aftereffect." The latter process may be the main basis for these interference effects.
\end{abstract}

The "kindling effect" has been investigated in a number of laboratories as a model of learning, a model of epilepsy, or as an example of behavioral change of interest in its own right (e.g., Gaito \& Gaito, 1974; Goddard, McIntyre, \& Leech, 1969; Racine, 1972; Wada \& Sato, 1975). Repeated lowlevel electrical stimulation of any of a number of brain regions induces progressive changes which culminate in clonic convulsions; as stimulation trials proceed, the animal's behavior changes in a gradual and predictable way from an initial stage of exploratory behavior (Stage 1), to behavioral automatisms usually involving facial contractions, chewing movements, eye closure, and salivation (Stage 2), and, finally, to a motor seizure (Stage 3). Behavioral, chemical, electrophysiological, and neurological aspects of this effect have been investigated by many researchers (Gaito, 1976a; Racine, 1978).

Once the motor seizure stage has been achieved, usually after 10-15 trials with rats, subsequent stimulation trials will induce the clonic convulsions (CC), even if weeks or months elapse between stimulations (Gaito, 1976b). Thus, a permanent brain change is thought to result from kindling (Goddard et al., 1969). Kindled seizures characteristically have a latency measurable in seconds between the onset of stimulation and the onset of the convulsion. They last for a number of seconds after the electrical stimulation is terminated. The effect is remarkably resistant to a number of experimental manipulations (Gaito, 1976a). Careful morphological analyses have indicated that the effect is not due to edema, ion deposition, or any form of damage to the stimulated tissue (Goddard et al., 1969; Goddard \& Douglas, 1975; Racine, 1978). Although the amygdaloid complex appears to be particularly sensitive to kindling, the effect may be obtained from a number of different brain regions, especially those within the limbic system (Goddard et al., 1969; Burnham, 1975).

There are three types of negative transfer or interference effects that have been observed within the kindling paradigm. These are: intrafrequency, alter- nating stimulation of opposite sides; intrafrequency, successive unilateral stimulation; interfrequency, successive unilateral or bilateral stimulation. The kindling effect is valuable as a model of learning or epilepsy or as an indicator of behavioral change, and the use of this behavioral paradigm could allow for the determination of brain events underlying the behavioral changes. Thus, these interference effects may be of great significance, because, presumably, a second set of brain events is interfering with those responsible for the development of kindling, or the original process is being modified. The purpose of this paper is to review research results concerning these three interference events.

\section{INTRAFREQUENCY, ALTERNATING STIMULATION OF OPPOSITE SIDES}

This type of interference is that involving successive stimulation of two homologous brain sites, for example, the amygdalae. If one amygdala is stimulated periodically with low intensity current, Stage 3 is usually achieved after an average of approximately 15 trials. If stimulation trials are maintained at that primary site until a criterion (e.g., six convulsions) is achieved and then the opposite amygdala is stimulated (secondary site), the criterion for the latter is achieved within a fewer number of trials, but increased latency to convulsion results (i.e., greater time between onset of stimulation and onset of convulsion). Thus, both positive and negative transfer occurs (Goddard et al., 1969; McIntyre \& Goddard, 1973). If the animal is now stimulated again at the primary site, it does not convulse on the first trial. A few trials are required to reach the convulsion stage, thus indicating negative transfer. Similar results have been reported by a number of researchers (e.g., Burnham, 1975).

Gaito (1976b) decided to extend the number of alternating stimulation phases to 10 or more (instead of just three) to determine the point where the transfer between hemispheres would disappear and the 
responses for both hemispheres would become approximately the same. The procedure involved three trials a day and stimulation at an intensity of $100 \mu \mathrm{A}$ for $30 \mathrm{sec}$ in each trial. Stimulation was maintained at one amygdala until six convulsions were observed, then applied at the contralateral amygdala until the same criterion was attained, then reinstated at the site first stimulated, and so on, in a pattern of sequential alternation of unilateral stimulation of the two amygdalae. Under these circumstances, instead of the expected stabilization of the responses, an "oscillation effect" developed (Gaito, 1976b). This effect consisted of the occurrence of consistently low values for one amygdala and consistently high values for the other as stimulation was alternated between the two. The effect was most prominent ir. latency data. This effect, presumably, shows consistent interference, expressed by the greater value in one site.

A number of variables which might affect this oscillation tendency were investigated. In latency data, simultaneous bilateral stimulation prior to, or after, the development of oscillation had no disruptive effect on the oscillation tendency (Gaito, 1976c). The tendency persisted with 1,3 , or 6 trials per day (Gaito, 1976d) and 1, 6, and 12 convulsions per phase (Gaito, 1976e). Oscillation was as prominent in old rats (420-475 days of age) as in younger ones (135-200 days) (Gaito \& Nobrega, 1977). Rest intervals of 1,3 , and 6 months interspersed between two sets of 10 sequential alternation phases had no deleterious effect (Gaito, 1976f). The tendency was unaffected by intensities of 100 and $280 \mu \mathrm{A}$, but $560 \mu \mathrm{A}$ intensity had a slight disruptive effect (Gaito, 1977a).

These results were obtained with intensities that were constant for both sides. However, if the threshold for a convulsion is determined for each side and these intensities are used in each of the 10 phases, the oscillation effect still persisted in latency data (Gaito, 1977b). Using this threshold method, a number of other variables were evaluated. The oscillation effect was present in latency data when the alternation from one side to the other occurred following a single convulsion trial (Gaito, 1978a), when a 5-sec duration of stimulation was used rather than $30 \mathrm{sec}$ (Gaito, 1978b), and when a 14-day interval was imposed between alternation phases (Gaito, 1978c). This effect has persisted over 50 phases, 25 for each side (Gaito, 1978d), or when the brain commissures are severed (Nobrega \& Gaito, 1978). However, an oscillation pattern does not result if each successive phase involves stimulation of the same unilateral structure (Gaito \& Nobrega, 1978).

Thus, it appears that the oscillation effect, or interference effect, is an extremely robust event for latency data. However, few cases of oscillation occur in criterion data (i.e., number of trials to six convul- sions). Each of the above manipulations disrupted partially, or completely, this weak oscillatory tendency. The duration of convulsion measure was not analyzed in these experiments because few rats showing an oscillation pattern were detected in the first study. When this measure was analyzed over a number of experiments, an oscillation pattern appeared which was stronger than that for criterion data but not as strong as for the latency measure (Gaito, Gaito, \& Nobrega, 1977).

There are two main types of oscillation patterns that occurred consistently in the Gaito et al. research: primary oscillation (low values for the primary site and high values for the secondary site, for at least 8 of the 10 phases) and secondary oscillation (the opposite pattern). There was a predominant tendency for increased latency to be present in the secondary site (primary oscillation) for most rats over all or most phases of stimulation (Gaito, Gaito, \& Nobrega, 1977; Gaito, Nobrega, \& Gaito, 1978).

Factor analysis of data for 139 rats over a number of experiments indicated the presence of two factors for latency data: a Primary Site Stimulation Factor and a Secondary Site Stimulation Factor (Gaito, Gaito, \& Nobrega, 1977). Later analyses with another 125 rats found the same results (Gaito \& Gaito, 1979). Stimulation of only the primary site over 10 phases produced a single factor (Gaito \& Gaito, 1979).

The oscillation effect (with predominance of primary oscillation) which Gaito et al. observed seemed to be an authentic event which was of an interference nature. That the oscillation effect is an authentic phenomenon was suggested also by the data of other researchers. McIntyre (1975) and McIntyre and Goddard (1973) reported latency values of primary oscillation nature for groups of rats over three phases of sequential alternation, that is, a "lowhigh-low" pattern. Individual data were not shown. Presumably, some rats with a secondary oscillation pattern were present, but primary oscillation predominated overall.

\section{INTRAFREQUENCY, SUCCESSIVE UNILATERAL STIMULATION}

Mucha and Pinel (1977) reported that repeated, periodic stimulation with $60-\mathrm{Hz}$ current had a decremental effect on both motor seizures and on specific brain wave patterns in the EEG. The effect on the class of motor seizure (or severity of convulsion) had dissipated by $20 \mathrm{~min}$. The duration of convulsion was reduced through a 45 -min period but was back to usual levels by $90 \mathrm{~min}$. This interference effect is a type of unilateral stimulation interference.

Other researchers (e.g., Gaito et al.) do not report these events, presumably because they are not looking for these aspects, or because their procedures 
are not suitable for detecting them. The Mucha and Pinel stimulation condition appears to be a sensitive means of detecting this type of interference.

Although Gaito et al. have not noted the type of interference reported by Mucha and Pinel, they observed another event that may be similar. With many convulsions (e.g., 60 or more), a number of rats stopped convulsing when the current was terminated after the beginning of a convulsion, whereas the typical response was to continue convulsing well beyond the offset point. This result may be similar to the Mucha and Pinel event in which duration of motor seizures was reduced up to $90 \mathrm{~min}$ after stimulation with $60 \mathrm{~Hz}$ current.

\section{INTERFREQUENCY, SUCCESSIVE UNILATERAL OR BILATERAL STIMULATION}

The previous interference effects occurred during the use of one specific frequency. However, recently Gaito has found that one frequency is able to interfere with the effect of another frequency.

Goddard et al. (1969) indicated that there was a reduced probability of eliciting a convulsion at a given intensity for frequencies above and below $60-\mathrm{Hz}$. Thus, Gaito thought it might be possible to find some frequencies other than $60-\mathrm{Hz}$ which could interfere with $60-\mathrm{Hz}$ brain stimulation results. With a few rats, a number of frequencies below and above $60-\mathrm{Hz}$ were evaluated (i.e., 30, 20, 15, 10, 5, 3, and $1 ; 100,150,200,300,400,500,1,000,2,000$, and $4,000)$. Stage 2 or 3 behavior was observed at all frequencies except 3 and $1 \mathrm{~Hz}$, although greater intensities were required to elicit these behaviors (Gaito, 1979b).

In this attempt to determine frequencies that might be used as potential interference agents, two criteria were used: (a) Stage 2 or Stage 3 behavior should not usually be elicited with low or moderate intensities (e.g., up to $560 \mu \mathrm{A}$ ); (b) no consistent convulsion pattern should be elicited even at higher intensities.

Only 3- and $1-\mathrm{Hz}$ stimulation met these criteria. Seldom did Stage 2 or Stage 3 behavior occur below an intensity of $560 \mu \mathrm{A}$; Stage 1 behavior was the typical response in almost all cases. Furthermore, although convulsions did occur at intensities greater than $560 \mu \mathrm{A}$, stable convulsion patterns on successive trials of stimulation did not occur. Thus, $3 \mathrm{~Hz}$ and, later, $1 \mathrm{~Hz}$ were evaluated as potential "interference agents."

In these research efforts, two types of experimental procedures were used: reversal experiment and suppression experiment. The former experiment involves using rats that have achieved a stable convulsion state (e.g., after 60 convulsion trials, three trials a day, one hour between trials). During the last six trials, the threshold for a convulsion is determined. Thirtysix trials of $1-$ or $3-\mathrm{Hz}$ stimulation are provided over 12 days at twice the threshold value. Then 30 test trials with $60-\mathrm{Hz}$ stimulation are used to determine if the rat will convulse at its previous threshold point.

The suppression experiment is appropriate with rats at any stage in the kindling process. The usual procedure is with rats at Stage 2 or Stage 3. These animals are given three trials a day. The first and third trials for experimental rats are $1-$ or $3-\mathrm{Hz}$ stimulation. On Trial 2 of each day, all rats receive stimulation with $60-\mathrm{Hz}$ sine waves. Thus, the suppressive effect of $1-$ or $3-\mathrm{Hz}$ stimulation on behavior induced by $60-\mathrm{Hz}$ stimulation can be evaluated on a day-by-day basis.

In a series of experiments, $3-\mathrm{Hz}$ stimulation consistently produced an interference effect, that is, suppression of convulsions initiated by $60-\mathrm{Hz}$ stimulation (Gaito, 1979b, 1979c; Gaito, Nobrega, \& Gaito, 1980). Another experiment evaluated the effect of varying durations of $1-\mathrm{Hz}$ stimulation, namely, 0 , $5,15,30,60,120,180$, and $600 \mathrm{sec}$ (Gaito, 1980). The 5-sec condition gave the same results as the control condition ( 0 -sec stimulation)-there was no interference effect. With $15 \mathrm{sec}$ of stimulation, there was a minor effect. The effect was more pronounced at $30 \mathrm{sec}$. The 60,120 , and $180 \mathrm{sec}$ of stimulation produced drastic effects. However, the greatest effect was with the 600 -sec stimulation period. The overall result was that of an increasing interference or suppression effect as duration of stimulation increased. Similar results occurred with $3-\mathrm{Hz}$ stimulation (Gaito, Note 1).

\section{BASIS FOR INTERFERENCE EFFECTS}

\section{Alternating Stimulation}

Gaito, Nobrega, and Gaito (1978) indicated that there are a number of possible bases for the oscillation effect, namely, chance aspects, differential thresholds for the two sides, differential placement of the two electrodes, differential effectiveness of the two electrodes, and differential natural reactivity of the two brain sites. Each of these has been systematically evaluated and eliminated as a basis for the oscillation effect (Gaito, 1979a; Gaito, Nobrega, \& Gaito, 1978).

Having eliminated these possible bases, it appeared that some type of interaction between the two brain sites probably was responsible for the effect. Transfer and interference effects between primary and secondary sites are presumed to be responsible for the results by McIntyre (1975) and McIntyre and Goddard (1973) over three phases of unilateral stimulation. Thus, it seems that some type of interaction between the two brain sites is occurring over the 10 
or more phases involved in the sequential alternation research.

Presumably, there are some inhibitory and/or facilitory effects from the primary site to the secondary site, and vice versa, to produce the interference effect (Goddard et al., 1969; McIntyre \& Goddard, 1973; Nobrega \& Gaito, 1978). However, it is not clear what the exact basis for the effect is. A pattern appears to be set up, either primary oscillation or secondary oscillation, during Phases 1 and 2, or by Phases 3 and 4 , and most rats continue with this pattern for the remainder of the 10 phases. In one experiment in which rats were stimulated through 50 phases, some rats showed a consistent pattern of oscillation for the 50 phases (Gaito, 1978d).

These interhemispheric interaction results appear to be consistent with the two-process interpretation of Goddard et al. (1969) and McIntyre and Goddard (1973). These individuals suggested the involvement of two effects in latency and criterion data. These are: (1) a long-term effect of a positive transfer nature, due to modified neural circuitry (Goddard et al., 1969; and (2) a short-term "aftereffect" of a negative transfer nature (McIntyre \& Goddard, 1973). The former would involve the establishment of two specific, but widespread, neural circuits. One would develop during stimulation of the primary site. During kindling of the secondary site, the second circuit would tie into response elements of the first circuit and trigger convulsions in fewer trials than were required with stimulation of the primary site. As a result of this utilization, however, the circuits in the primary site would be changed to correspond with the activity of the second region and lose some of their correspondence with the original ones. A few trials would be necessary to reestablish the original pattern when stimulating the primary site again. The "aftereffect" would result from convulsions in both primary and secondary sites; its exact nature and mechanism are not known. The "aftereffect" was able to suppress seizure activity in different parts of the nervous system, was proportional to the number of convulsions, and spontaneously dissipated over time; complete dissipation occurred in two weeks or more (McIntyre \& Goddard, 1973). These two processes would be operating on one side or on two sides, depending on the stimulation conditions.

Burnham (1975) also suggested that two mechanisms were involved in these kindling events. One was a convulsion mechanism that had duration as its indicator; the other was a triggering mechanism that was expressed in terms of latency. This classification was based on the fact that latency and duration results, over three sequential alternation phases, were distinctively different. Duration values remained approximately the same for each phase, whereas latencies slowly decreased over phases and within phases. Again, these two aspects would operate on one or two sides.
Nobrega and Gaito (1978) discussed facilitation and interference effects with normal and split-brain rats. They indicated that interference effects were via hemispheric commissures and the brain stem. Their ideas would be consistent with the two-effect idea by Goddard et al. and McIntyre and Goddard.

\section{Successive Unilateral Stimulation}

Presumably, the interference pattern set up in this case is similar to that observed in the successive stimulation of homologous structures. Postseizure inhibition processes develop during stimulation and persist for a period of time. If stimulation occurs within the period that this inhibition process is functional, there is a reduced probability for the occurrence of a convulsion at its usual strength and duration (Goddard et al., 1969; Mucha \& Pinel, 1977; Racine, 1972).

\section{Interfrequency Stimulation}

The exact basis for the $1-\mathrm{Hz}$ and $3-\mathrm{Hz}$ interference effect is not clear at this time, but the inhibitory process may be similar to that observed in the other two interference cases. The results appear not to be due to damage at the site of stimulation. Stimulation with $60-\mathrm{Hz}$ current at low and moderate intensities does not produce damage (Goddard et al., 1969; Goddard \& Douglas, 1975; Racine, 1978). Thus, there is no reason to expect that stimulation with $1-\mathrm{Hz}$ or $3-\mathrm{Hz}$ current at the same intensities as those used with $60-\mathrm{Hz}$ stimulation should produce damage. Furthermore, in the Gaito experiments, histological examinations indicated no observable gross lesions; the tissue around electrode tips for rats stimulated with $60-\mathrm{Hz}$ current appeared similar to that of rats stimulated with $3-\mathrm{Hz}$ or $1-\mathrm{Hz}$ current. Likewise, if lesions were the basis for the interference effect, the rats would not convulse again at previous threshold levels (Goddard et al., 1969; Racine, 1978). However, the results indicated that most rats convulse at previous low thresholds after 15-16 days of rest (Gaito, 1980). Furthermore, with intertrial intervals of 1 and $3 \mathrm{~h}$, the suppression effect is pronounced. However, when the interval is increased to $24 \mathrm{~h}$, the suppression effect is present but at a greatly reduced level; apparently, some of the effect dissipates within this time period (research underway).

The last two points, that most rats convulse at previous ETI levels after a rest of 15 or 16 days and that a 24-h intertrial interval reduces the magnitude of the suppression effect, are indications that the suppression effect is a relatively transient event, possibly similar to the "aftereffect" of McIntyre and Goddard (1973), and contrasts with the kindling effect. The latter persists for months or longer (Goddard et al., 1969; Racine, 1978).

There are two possible interpretations of these results. The first is that there are two brain processes involved in overall kindling phenomena. There is 
Process $\mathrm{A}$ that is developed during kindling and possibly involves circuitry and/or synaptic changes to produce a long-term effect. This would be the Goddard et al. (1969) effect which provides longterm stability. Another brain event, Process B, would develop during the stimulation with 1 - or $3-\mathrm{Hz}$. This process would be antagonistic to Process $A$ and produce the suppression effect. This is a two-process interpretation with Process B dissipating over time; the two processes are separate but interacting.

A second possibility is that the kindling procedures develop the relatively permanent Process $A$, and this process would be modified by stimulation with 1 - and 3- $\mathrm{Hz}$ current to bring about an interference effect. However, some or all of these modifications would decay over time and Process $A$ would remain relatively intact as Process $\mathbf{A}^{\prime}$. This is a two-process interpretation with Process $B$ being superimposed on Process $\mathbf{A}$ at one site to become Process $\mathbf{A}^{\prime}$.

Most of the data are consistent with both of these possibilities. Obviously, further research is required to differentiate between these possibilities.

\section{Summarizing Comments}

There are both interfrequency and intrafrequency interference effects within the kindling paradigm. The interference effects occur during unilateral and alternating stimulation events. In the alternating stimulation case, stimulation of one brain site sets up interference effects to later stimulation of the homologous brain site. This type of interference effect is assumed to result because of inhibitory effects of the one brain site on the other (McIntyre \& Goddard, 1973; Nobrega \& Gaito, 1978), probably via interhemispheric commissures, and/or brain stem connections (Racine, 1978). The other intrafrequency interference result with successive unilateral stimulation is probably due to the same type of process, that is, an inhibitory process developing with periodic stimulation. Thus, stimulation at the primary site may set up an inhibitory process relative to later stimulation of the homologous site in the opposite hemisphere as well as an inhibitory process developing with periodic stimulation. Thus, stimulation at the primary site may set up an inhibitory process relative to later stimulation of the homologous site in the opposite hemisphere as well as an inhibitory process at the primary site which would interfere with later stimulation of the primary site. The McIntyre and Goddard and the Mucha and Pinel results are consistent with this interpretation. Presumably, the results with interfrequency stimulation are due also to an inhibitory or interference process, but one that is elicited by a frequency different from that which developed the convulsive state.

There have been a number of suggestions as to the mechanisms involved in the alternating stimulation case, as discussed above. Goddard et al. (1969) and McIntyre and Goddard (1973) suggested the involvement of two effects operating to produce specific results in latency and criterion data. These are the modified neural circuitry (Goddard et al., 1969) and the short-term "aftereffect" (McIntyre \& Goddard, 1973). Burnham (1975) also suggested two mechanisms, a convulsion mechanism and a triggering mechanism.

The factor analysis results of Gaito et al. (1977) also are relevant to mechanism aspects. They found two factors in latency and criterion data and one factor for duration data. These results of two factors in the latency and criterion measures may appear somewhat different from the two factors (or effects) of Goddard et al. (1969) and McIntyre and Goddard (1973). Their first factor was a positive transfer one of a long-term nature, and the "aftereffect"' (second factor) was a less durable one of a negative transfer nature. However, the "aftereffect" would be involved in convulsions from stimulation of both the primary and secondary sites. Thus, the two factors can be viewed as (a) a primary site factor of a neurological trace nature with a residual "aftereffect", and (b) a secondary site factor of a neurological trace nature with a residual "aftereffect." Thus, the two factors of Gaito et al. are basically the same as the two factors of Goddard et al. and McIntyre \& Goddard (Table 1 ).

If one considers the factor structure over the three dependent variables, there are three factors (Table 1). One single factor would be the convulsion mechanism of Burnham, inasmuch as duration is the indicator of this mechanism (Burnham, 1975). The

Table 1

Factors or Mechanisms Present During Sequential Alternation Within the Kindling Paradigm

\begin{tabular}{|c|c|c|c|}
\hline $\begin{array}{c}\text { Goddard et al. (1969) } \\
\text { McIntyre-Goddard (1973) } \\
\text { Nobrega-Gaito (1978) }\end{array}$ & Burnham (1975) & $\begin{array}{l}\text { Factor Analyses: } \\
\text { Gaito et al. (1977) } \\
\end{array}$ & Measures as Indicators \\
\hline $\begin{array}{l}\text { Neurological trace with } \\
\text { aftereffect (primary site) }\end{array}$ & $\begin{array}{l}\text { Triggering mechanism } \\
\text { (primary site) }\end{array}$ & $\begin{array}{l}\text { Primary site } \\
\text { stimulation factor }\end{array}$ & Latency, criterion \\
\hline \multirow[t]{2}{*}{$\begin{array}{l}\text { Neurological trace with } \\
\text { aftereffect (secondary site) }\end{array}$} & $\begin{array}{l}\text { Triggering mechanism } \\
\text { (secondary site) }\end{array}$ & $\begin{array}{l}\text { Secondary site } \\
\text { stimulation factor }\end{array}$ & Latency, criterion \\
\hline & $\begin{array}{l}\text { Convulsion mechanism } \\
\text { (both sites) }\end{array}$ & Duration factor & Duration \\
\hline
\end{tabular}


Gaito et al. two factors in latency and criterion data would be related to the Burnham triggering mechanism, a triggering mechanism for each of the sides (Table 1), and to the neurological trace and "aftereffect of Goddard et al. and McIntyre and Goddard.

Thus, once can provide a summary of kindling events from Table 1 by the following statement. There is a "neurological trace" for both the primary and secondary sites (Primary Site Stimulation Factor, Secondary Site Stimulation Factor). This is the main mechanism developed during kindling and probably has neural circuitry changes involving the synapse (Goddard \& Douglas, 1975). Another mechanism, "aftereffect," determines the availability of the "neurological trace" for actual operation. Presumably, the interfrequency interference effect observed by Gaito et al. and the Mucha and Pinel interference effects may be the "aftereffect" of McIntyre and Goddard (which is assumed to produce interference). The "aftereffect" dissipates within 2 weeks; the increased ETI aspect in the interfrequency situation is lost for most animals in 15 or 16 days (i.e., most rats are convulsing again at previous ETI values). The third factor, Burnham's convulsion mechanism for each side, indicates the interaction of the first two factors and is expressed as the duration of the convulsion state.

\section{REFERENCE NOTE}

1. Gaito, J. The effect of varying durations of stimulation on the $3-\mathrm{Hz}$ interference effect. Submitted for publication, 1979. (e)

\section{REFERENCES}

Burnham, W. M. Primary and "transfer" seizure development in the kindled rat. Canadian Journal of Neurological Sciences, $1975,2,417-428$.

Gaito, J. The kindling effect as a model of epilepsy. Psychological Bulletin, 1976, 83, 1097-1109. (a)

Gaito, J. An oscillation effect during sequential alternations of unilateral amygdaloid stimulations within the kindling paradigm. Physiological Psychology, 1976, 4, 303-306. (b)

GaIto, J. The effect of bilateral stimulation during sequential alternation of unilateral amygdaloid stimulation. Bulletin of the Psychonomic Society, 1976, 4, 355-357. (c)

Gaito, J. The effect of number of trials per day during sequential alternation of unilateral amygdaloid stimulation. Bulletin of the Psychonomic Society, 1976, 4, 403-404. (d)

GaIto, J. The effect of number of convulsions per phase on the oscillation tendency. Bulletin of the Psychonomic Society, 1976, 8, 392-394. (e)

Gaito, J. The effect of varying rest intervals following the development of oscillation during amygdaloid stimulation. Bulletin of the Psychonomic Society, 1976, 8, 457-458. (f)

Garto, J. The effect of inensity during sequential alternation of unilateral amygdaloid stimulation. Bulletin of the Psychonomic Society, 1977, 9, 64-66. (a)

GaITo, J. The oscillation effect at near-threshold intensities during sequential alternation of unilateral amygdaloid stimulation. Bulletin of the Psychonomic Society, 1977, 10, 145-148. (b)

Gaito, J. Trial-by-trial alternation of unilateral amygdaloid stimulation. Bulletin of the Psychonomic Society, 1978, 12, 47-49. (a)
Gaito, J. An oscillation effect at 5 seconds duration of stimulation. Bulletin of the Psychonomic Society, 1978, 12, 323324. (b)

Gaito, J. The effect of 14-day interval on the oscillation effect during sequential alternation of unilateral amygdaloid stimulation. Bulletin of the Psychonomic Society, 1978, 12, 113. 116. (c)

Gairo, J. The oscillation effect over long-term periods. Bulletin of the Psychonomic Society, 1978, 11, 9-12. (d)

Gaito, J. An experimental evaluation of differential natural reactivity as a basis for the oscillation effect. Bulletin of the Psychonomic Society, 1979, 13, 53-54. (a)

GaITo, J. Three $\mathrm{Hz}$ brain stimulation interferes with various aspects of the kindling effect. Bulletin of the Psychonomic Society, 1979, 13, 67-70. (b)

Gaito, J. Suppression of $60 \mathrm{~Hz}$ induced convulsive behavior by $3 \mathrm{~Hz}$ brain stimulation. Bulletin of the Psychonomic Society, 1979, 13, 223-226. (c)

Gaito, J. The effect of varying durations of stimulation on the $1 \mathrm{~Hz}$ interference effect. Canadian Journal of Neurological Sciences, 1980 , in press.

Gaito, J., \& Gaito, S. T. Interanimal negative transfer of the kindling effect. Physiological Psychology, 1974, 2, 379-382.

Gaito, J., \& Gaito, S. T. A factor analysis of data from sequential alternation of amygdaloid stimulation: A replication. Physiological Psychology, 1979, 7, 437-443.

Gaito, J., Gaito, S. T., \& Nobrega, J. N. A factor analysis of data from ten phases of sequential alternations of amygdaloid stimulation within the kindling paradigm. Physiological Psychology, 1977, 5, 300-310.

Gaito, J., \& Nobrega, J. The oscillation effect during sequential alternation of amygdaloid stimulation with aged rats. Bulletin of the Psychonomic Society, 1977, 9, 151-154.

Gaito, J., \& Nobrega, J. N. Random oscillation patterns with stimulation of a single brain site. Bulletin of the Psychonomic Society, 1978, 11, 65-67.

Gaito, J., Nobrega, J. N., \& Gaito, S. T. Statistical evaluation of several aspects concerning the oscillation effect. Physiological Psychology, 1978, 6, 209-214.

Gaito, J., Nobrega, J. N., \& Gaito, S. T. Interference effect of $3 \mathrm{~Hz}$ brain stimulation on kindling behavior induced by $60 \mathrm{~Hz}$ stimulation. Epilepsia, 1980, 21, 73-84.

GoddarD, G. V., \& Douglas, R. M. Does the engram of kindling model the engram of normal long term memory? Canadian Journal of Neurological Sciences, 1975, 2, 385-394.

Goddard, G. V., McIntyre, D. C., \& Leech, C. K. A permanent change in brain function resulting from daily electrical stimulation. Experimental Neurology, 1969, 25, 295-330.

MyInTyRe, D. C. Split-brain rat: Transfer and interference of kindled amygdala convulsions. Canadian Journal of Neurological Sciences, 1975, 2, 429-437.

MCIntyre, D. C., \& GoddaRd, G. V. Transfer, interference and spontaneous recovery of convulsions kindled from the rat amygdala. Electroencephalography and Clinical Neurophysiology, 1973, 35, 533-543.

Mucha, R. F., \& Pinel, J. P. J. Postseizure inhibition of kindled seizure. Experimental Neurology, 1977, 54, 266-282.

Nobrega, J. N., \& Gaito, J. Long term induction of kindled seizures in rats: Interhemispheric factors. Canadian Journal of Neurological Sciences, 1978, 5, 223-230.

RACINE, R. Kindling: The first decade. Neurosurgery, 1978, 3, 234-252.

RACINE, R. J. Modification of seizure activity by electrical stimulation: I. After-discharge threshold. Electroencephalography and Clinical Neurophysiology, 1972, 32, 269-279.

WadA, J. A., \& SAto, M. The generalized convulsive seizure state induced by daily electrical stimulation of the amygdala in split brain cats. Epilepsia, 1975, 16, 417-430.

(Received for publication November 7, 1979; revision accepted December 17, 1979.) 